\title{
Synthesis and Mesomorphic Properties of New Swallow-tailed Liquid Crystals Derived from 1,3-Dialkoxy-2-propanols
}

\author{
Kyung-Tae Kang, ${ }^{*}$ Seng Kue Lee, Chang Won Park, Sang Hui Cho, Jong Gun Lee, \\ Soon-Kyu Choi, and Yong Bae Kim \\ Department of Chemistry and Chemistry Institute for Functional Materials, Pusan National University, Busan 609-735, Korea \\ "E-mail: totkang@pusan.ackr. \\ "Department of Chemistry, Dong-A University, Busan 604-714, Korea \\ ${ }^{*}$ Liquid Crystal Research Center and Department of Chemistry, Konkuk University, Seoul 143-710, Korea \\ Received May 19, 2006
}

\begin{abstract}
New liquid-crystalline biphenyl carboxylates with an achiral swallow-tail derived from 1,3-dialkoxy-2propanol [ $\left.(\mathrm{ROCII})_{2} \mathrm{CIIOI}\right]$ where $\mathrm{R}$ is methyl, ethyl, propyl, butyl, $\mathrm{CII}_{2} \mathrm{CF}_{3}$, and $\mathrm{CII}_{2} \mathrm{CF}_{2} \mathrm{CF}_{3}$ were prepared. These achiral liquid crystals having 1,3-dialkoxy-2-propyl moieties exhibit diverse phase sequences [I- $\mathrm{m} \Lambda$ $(\mathrm{SmC})-(\mathrm{SmCalt})-\mathrm{Cr}]$ depending on the substituent $\mathrm{R}$ group of the swallow-tail. The compounds carrying a fluorinated swallow-tail exhibit antiferroelectric-like smectic $\mathrm{C}$ phases, and their temperature ranges are broader than the corresponding non-fluorinated swallow-tailed ones.
\end{abstract}

Key Words : Liquid crystal, Swallow-tailed liquid crystal, Antiferroelectric-like phase, Fluorinated alcohols, 1,3-Dialkoxy-2-propanols

\section{Introduction}

Ferroelecricity ${ }^{1}$ and antiferroelectricity ${ }^{2}$ in liquid crystals were usually observed in the chiral molecules. However, achiral swallow-tailed compounds have been demonstrated to display 'antiferroelectric-like' phase, so-called SmCalt phase and can be used as a host component of antiferroelectric mixture. ${ }^{3}$ Achiral bent-core mesogens can also form polar smectic layers and exhibit ferroelectric and antiferroelectric behaviors. ${ }^{4}$ As the basic difference between the ferroelectric and antiferroelectric orderings is the tilting direction between adjacent smectic layers, the interlayer interaction plays a key role in determining the clinicity. Thus the structure of the peripheral part existing near the interfaces between the smectic layers gave a significant and sensitive effect on the interlayer interaction. For example, while 1-propylbutyl swallow-tailed biphenyl carboxylate A exibits antiferroelectric ordering, the related ring-tailed compounds show ferroelectric ordering. ${ }^{3 b}$ Moreover, the odd-even effect of the peripheral alkyl chain was observed in the achiral non-branched rod-shaped liquid crystals ${ }^{5}$ and in the banana shaped mesogens with chiral terminal chains. ${ }^{6}$ It is important to investigate the relationship between chemical structure and mesomorphic properties for a series of liquid crystals with a systematic structural variation. However, in all the achiral or chiral ${ }^{7}$ swallow-tailed compounds, the variation of swallow-tailed moieties has been limited to branched alkyl groups derived from alcohols $\left[\left(\mathrm{RCH}_{2} \mathrm{CH}_{2}\right)_{2}-\right.$ $\mathrm{CHOH}$ ] where $\mathrm{R}$ is a simple alkyl. However, replacement of two methylene groups with oxygen atoms produces alcohols $\left[\left(\mathrm{ROCH}_{2}\right)_{2} \mathrm{CHOH}\right]$ where structural variation of $\mathrm{R}$ is practically easier from the synthetic point of view. The substituent $\mathrm{R}$ can be varied from simple alkyl groups (Me, $\mathrm{Et}, \mathrm{Pr}$, and $\mathrm{Bu}$ ) and even to fluorinated alkyl chains such as
$\mathrm{CH}_{2} \mathrm{CF}_{3}$ and $\mathrm{CH}_{2} \mathrm{CF}_{2} \mathrm{CF}_{3}$. In this regard, we report herein the synthesis of new achiral swallow-tailed compounds derived from 1,3-dialkoxy-2-propanols and the investigations of their mesomorphic properties.

\section{Experimental Section}

'H-NMR spectra were recorded on Varian Gemini-200 $(200 \mathrm{MHz})$ and Varian Inova $(500 \mathrm{MHz})$ spectrometer using chloroform as an internal standard. The latter instrument was also used for recording ${ }^{13} \mathrm{C}$ NMR spectra in $\mathrm{CDCl}_{3}$ (solvent and internal reference). Elemental analyses were performed at the National Center for Inter-University Research Facilities, Seoul National University. Phase transition temperature and phase appearance of final products were measured using polarizing microscope (Olympus BH-2) with a hot stage and a controller (Mettler FP-800-HT heating stage). Transition temperature and enthalpy were determined by differential scanning calorimetry (DSC) using a Perkin-Elmer DSC-7 calorimeter. Debenzylation of benzyl ethers to the corresponding alcohols and phenols were carried out in the Parr hydrogenation reactor (Parr 3916EKX).

Preparation of 1,3-dialkoxy-2-propanols 3. To the sodium methoxide solution prepared from a freshly distilled methanol $(47 \mathrm{~mL})$ and metallic sodium $(8.8 \mathrm{~g}, 0.38 \mathrm{~mol})$ was added epichlorohydrin $(\mathbf{1}, 17.6 \mathrm{~g}, 0.19 \mathrm{~mol})$. The mixture was refluxed for $24 \mathrm{~h}$ under an argon atmosphere. The methanol was evaporated and the residue was distilled under reduced pressure [bp. $78-81^{\circ} \mathrm{C} / 20 \mathrm{mmHg}$ ] to give $9.80 \mathrm{~g}$ (43\%) of 1,3-dimethoxy-2-propanol (3a). Compounds $\mathbf{3 b}$-3f were similarly prepared in $77,70,56,85$, and $95 \%$ yields, respectively.

3a: 'H NMR $\delta 2.25$ (br s), 3.31 (s, 6H), 3.34-3.44 (m, 4H), 3.87-3.99 (m, 1H). 3b: bp. $98-101^{\circ} \mathrm{C} / 20 \mathrm{mmHg}{ }^{1} \mathrm{H}$ NMR $\delta$ 
$1.12(\mathrm{t}, 6 \mathrm{H}, J=7.0 \mathrm{~Hz}), 2.81(\mathrm{~s}, 1 \mathrm{H}), 3.31-3.50(\mathrm{~m}, 8 \mathrm{H})$, 3.83-3.89 (m, 1H). 3c: bp. $65-67^{\circ} \mathrm{C} / 9 \mathrm{mmHg}{ }^{1} \mathrm{H}$ NMR $\delta$ $0.89(\mathrm{t}, 6 \mathrm{H}, J=7.2 \mathrm{~Hz}), 1.48-1.62(\mathrm{~m}, 4 \mathrm{H}), 2.15(\mathrm{br} \mathrm{s}), 3.37-$ $3.60(\mathrm{~m}, 8 \mathrm{H}), 3.89-3.95(\mathrm{~m}, 1 \mathrm{H}) .3 \mathrm{3d}:$ bp. $85-88^{\circ} \mathrm{C} / 9 \mathrm{mmHg}$ 'H NMR $\delta 0.87(\mathrm{t}, 6 \mathrm{H}, J=7.2 \mathrm{~Hz}), 1.22-1.40(\mathrm{~m}, 4 \mathrm{H}), 1.42-$ $1.59(\mathrm{~m}, 4 \mathrm{H}), 2.37$ (br s), 3.34-3.62 (m, 8H), 3.84-3.92 (m, $1 \mathrm{H})$. 3e: bp. $42-45^{\circ} \mathrm{C} / 9 \mathrm{mmHg}$ 'H NMR $\delta 2.37$ (br s), 3.65$3.97(\mathrm{~m}, 5 \mathrm{H}), 3.87$ (q, $4 \mathrm{H}, J=8.8 \mathrm{~Hz}$ ). 3f: bp. $62-64^{\circ} \mathrm{C} / 9$ mmHg ${ }^{1} \mathrm{H}$ NMR $\delta 2.24$ (br s), 3.68 (d, $4 \mathrm{H}, J=4.4 \mathrm{~Hz}$ ), 3.96 (t, $4 \mathrm{H}, J=13.0 \mathrm{~Hz}), 3.90-3.98(\mathrm{~m}, 1 \mathrm{H})$.

Preparation of 1,3-dialkoxy-2-propyl 4-benzyloxybenzoates 5. To a solution of 1,3-dimethoxy-2-propanol (3a, $1.63 \mathrm{~g}, 13.6 \mathrm{mmol})$ in $\mathrm{CH}_{2} \mathrm{Cl}_{2}(40 \mathrm{~mL})$ was added 1,3-dicyclohexylcarbodiimide (DCC, $3.07 \mathrm{~g}, 14.9 \mathrm{mmol}$ ), 4-dimethylaminopyridine (DMAP, $1.82 \mathrm{~g}, 14.9 \mathrm{mmol}$ ) and 4-benzyloxybenzoic acid $(4,2.80 \mathrm{~g}, 12.4 \mathrm{mmol})$. The mixture was stirred at room temperature ovenight. After filtration to remove precipitated materials, the filtrate was washed with $5 \%$ aq acetic acid $(20 \mathrm{~mL}), 5 \%$ aq sodium hydroxide $(20 \mathrm{~mL})$ and water, and then dried over $\mathrm{Na}_{2} \mathrm{SO}_{4}$. After evaporation of the solvent the crude product was chromatographed on silica gel (hexanes/ether $1: 1, R_{\mathrm{f}}=0.8$ ) to afford $3.78 \mathrm{~g}(93 \%)$ of $\mathbf{5 a}$. Compounds $\mathbf{5 b - 5 f}$ were similarly prepared in $63,62,60,86$, and $97 \%$ yields, respectively.

5a: ${ }^{1} \mathrm{H}$ NMR $\delta 3.38(\mathrm{~s}, 6 \mathrm{H}), 3.65(\mathrm{~d}, 4 \mathrm{H}, J=5.1 \mathrm{~Hz}), 5.11$ (s, 2H), 5.34 (quin, $1 \mathrm{H}, J=5.1 \mathrm{~Hz}$ ), 6.94-8.03 (m, 9H). 5b: 'H NMR $\delta 1.17(\mathrm{t}, 6 \mathrm{H}, J=7.0 \mathrm{~Hz}), 3.49-3.59(\mathrm{~m}, 4 \mathrm{H}), 3.69$ (d, $4 \mathrm{H}, J=5.1 \mathrm{~Hz}$ ), 5.11 (s, 2H), 5.30 (quin, $1 \mathrm{H}, J=5.1 \mathrm{~Hz}$ ), 6.94-8.03 (m, 9H). 5c: 'H NMR $\delta 0.97(\mathrm{t}, 6 \mathrm{H}, J=7.0 \mathrm{~Hz})$, 1.61 (sext, $4 \mathrm{H}, J=7.3 \mathrm{~Hz}), 3.37-3.48(\mathrm{~m}, 4 \mathrm{H}), 3.68(\mathrm{~d}, 4 \mathrm{H}, J$ $=5.1 \mathrm{~Hz}$ ), $5.11(\mathrm{~s}, 2 \mathrm{H}), 5.32$ (quin, $1 \mathrm{H}, J=5.1 \mathrm{~Hz}$ ), 6.94$8.03(\mathrm{~m}, 9 \mathrm{H}) .5 \mathrm{~d}$ : 'H NMR $\delta 0.87(\mathrm{t}, 6 \mathrm{H}, J=7.0 \mathrm{~Hz}), 1.30-$ $1.40(\mathrm{~m}, 4 \mathrm{H}), 1.41-1.58(\mathrm{~m}, 4 \mathrm{H}), 3.40-3.55(\mathrm{~m}, 4 \mathrm{H}), 3.66(\mathrm{~d}$, $4 \mathrm{H}, J=5.1 \mathrm{~Hz}$ ), $5.11(\mathrm{~s}, 2 \mathrm{H}), 5.29$ (quin, $1 \mathrm{H}, J=5.1 \mathrm{~Hz}$ ), 6.94-8.02 (m, 9H). 5e: ${ }^{1} \mathrm{H}$ NMR $\delta 3.80-3.97(\mathrm{~m}, 8 \mathrm{H}), 5.12$ (s, 2H), 5.29 (quin, $1 \mathrm{H}, J=5.1 \mathrm{~Hz}$ ), 6.97-8.03 (m, 9H). 5f: 'H NMR $\delta 3.86-4.01(\mathrm{~m}, 8 \mathrm{H}), 5.12(\mathrm{~s}, 2 \mathrm{H}), 5.30$ (quin, $1 \mathrm{H}, J$ $=5.1 \mathrm{~Hz}), 6.97-7.99(\mathrm{~m}, 9 \mathrm{H})$.

Preparation of 1,3-dialkoxy-2-propyl 4-hydroxybenzoates 6. A methanol $(18 \mathrm{~mL})$ solution of $5 \mathrm{a}(3.50 \mathrm{~g}, 10.6$ mmol) was shaken with a Paar hydrogenation reactor in the presence of $0.3 \mathrm{~g}$ of palladium catalyst $(5 \% \mathrm{Pd} / \mathrm{C})$ at $15 \mathrm{psi}$ pressure over a period of $3 \mathrm{~h}$. The catalyst was filtered off and the filtrate was dried over $\mathrm{Na}_{2} \mathrm{SO}_{4}$, and concentrated to give $2.50 \mathrm{~g}(98 \%)$ of $6 \mathrm{a}$. Compounds $6 \mathrm{~b}-6 \mathrm{f}$ were similarly prepared in $98,98,76,98$, and $97 \%$ yields, respectively.

6a: 'H NMR $\delta 3.40(\mathrm{~s}, 6 \mathrm{H}), 3.60-3.75(\mathrm{~m}, 4 \mathrm{H}), 5.37$ (quin, $1 \mathrm{H}, J=5.1 \mathrm{~Hz}), 6.72(\mathrm{~d}, 2 \mathrm{H}, J=8.8 \mathrm{~Hz}), 7.25(\mathrm{br} \mathrm{s}), 7.84(\mathrm{~d}$, $2 \mathrm{H}, J=8.8 \mathrm{~Hz}), 6 \mathrm{~b}:{ }^{1} \mathrm{H}$ NMR $\delta 1.18(\mathrm{t}, 6 \mathrm{H}, J=6.9 \mathrm{~Hz})$, $3.48-3.70(\mathrm{~m}, 4 \mathrm{H}), 3.74(\mathrm{~d}, 4 \mathrm{H}, J=5.1 \mathrm{~Hz}), 5.36$ (quin, $1 \mathrm{H}$, $J=5.1 \mathrm{~Hz}), 6.70$ (d, $2 \mathrm{H}, J=8.8 \mathrm{~Hz}), 7.0$ (br s), 7.79 (d, $2 \mathrm{H}$, $J=8.8 \mathrm{~Hz}) .6 \mathrm{c}:$ 'H NMR $\delta 0.87(\mathrm{t}, 6 \mathrm{H}, J=7.4 \mathrm{~Hz}), 1.58$ (sext, 4H, $J=7.4 \mathrm{~Hz}$ ), 3.40-3.51 (m, 4H), 3.72 (d, 4H, $J=$ $5.1 \mathrm{~Hz}$ ), 5.34 (quin, $1 \mathrm{H}, J=5.1 \mathrm{~Hz}), 6.73$ (d, $2 \mathrm{H}, J=8.8$ $\mathrm{Hz}), 7.25$ (br s), 7.81 (d, $2 \mathrm{H}, J=8.8 \mathrm{~Hz}$ ). 6d: 'H NMR $\delta 0.88$ (t, $6 \mathrm{H}, J=7.2 \mathrm{~Hz}$ ), 1.33 (sext, $4 \mathrm{H}, J=7.2 \mathrm{~Hz}$ ), 1.54 (quin,
$4 \mathrm{H}, J=7.2 \mathrm{~Hz}), 3.43-3.53(\mathrm{~m}, 4 \mathrm{H}), 3.69(\mathrm{~d}, 4 \mathrm{H}, J=5.1 \mathrm{~Hz})$, 5.32 (quin, $1 \mathrm{H}, J=5.1 \mathrm{~Hz}), 6.77(\mathrm{~d}, 2 \mathrm{H}, J=8.8 \mathrm{~Hz}), 7.30(\mathrm{br}$ s), 7.88 (d, $2 \mathrm{H}, J=8.8 \mathrm{~Hz}$ ). 6e: ${ }^{1} \mathrm{H}$ NMR $\delta 3.81-4.01(\mathrm{~m}$, $8 \mathrm{H}$ ), 5.33 (quin, $1 \mathrm{H}, J=5.1 \mathrm{~Hz}$ ), 6.40 (br s), 6.88 (d, $2 \mathrm{H}, J=$ $8.8 \mathrm{~Hz}$ ), 7.94 (d, $2 \mathrm{H}, J=8.8 \mathrm{~Hz}$ ). 6f: 'H NMR $\delta 3.86-3.95$ (m, 8H), 4.01 (br s), 5.28 (quin, $1 \mathrm{H}, J=5.1 \mathrm{~Hz}$ ), 6.88 (d, $2 \mathrm{H}$, $J=8.8 \mathrm{~Hz}), 7.93(\mathrm{~d}, 2 \mathrm{H}, J=8.8 \mathrm{~Hz})$.

Preparation of 4-[(1,3-dialkoxy-2-propyloxy)carbonyl] phenyl 4'-alkyloxy-(1,1'-biphenyl)-4-carboxylate 8 . To a solution of $6 \mathrm{a}(0.53 \mathrm{~g}, 2.21 \mathrm{mmol})$ in $\mathrm{CH}_{2} \mathrm{Cl}_{2}(15 \mathrm{~mL})$ was added DCC $(0.55 \mathrm{~g}, 2.65 \mathrm{mmol})$, DMAP $(0.32 \mathrm{~g}, 2.65$ mmol), and 4'-octyloxy-(1,1'-biphenyl)-4-carboxylic acid $(7 \mathrm{a}, 0.72 \mathrm{~g}, 2.21 \mathrm{mmol})$. The mixture was stirred at room temperature ovemight. After removal of precipitated materials, the filtrate was washed with $5 \%$ acetic acid $(20 \mathrm{~mL}), 5 \%$ aq sodium hydroxide $(20 \mathrm{~mL})$ and water, and then dried over $\mathrm{Na}_{2} \mathrm{SO}_{4}$. The concentrated residue was purified by column chromatography (silica gel, hexanes/ether $1: 1, \mathrm{R}_{\mathrm{f}}=0.6$ ) and followed by recrystalization from hexane to give $1.09 \mathrm{~g}$ $(90 \%)$ of $\mathbf{8 a}$. Compounds $\mathbf{8 b - 8 y}$ were similarly prepared.

8a (8C-Me): 'H-NMR $\delta 0.89$ (t, 3H, $J=6.6 \mathrm{~Hz}), 1.30-$ $1.85(\mathrm{~m}, 12 \mathrm{H}), 3.40(\mathrm{~s}, 6 \mathrm{H}), 3.68(\mathrm{~d}, 4 \mathrm{H}, J=5.1 \mathrm{~Hz}), 4.01(\mathrm{t}$, $2 \mathrm{H}, J=6.6 \mathrm{~Hz}$ ), 5.39 (quin, $1 \mathrm{H}, J=5.1 \mathrm{~Hz}), 7.00(\mathrm{~d}, 2 \mathrm{H}, J=$ $8.8 \mathrm{~Hz}), 7.31(\mathrm{~d}, 2 \mathrm{H}, J=8.8 \mathrm{~Hz}), 7.60(\mathrm{~d}, 2 \mathrm{H}, J=8.8 \mathrm{~Hz})$, $7.70(\mathrm{~d}, 2 \mathrm{H}, J=8.4 \mathrm{~Hz}), 8.16(\mathrm{~d}, 2 \mathrm{H}, J=8.8 \mathrm{~Hz}), 8.22(\mathrm{~d}$, $2 \mathrm{H}, J=8.4 \mathrm{~Hz}$ ); ${ }^{13} \mathrm{C}-\mathrm{NMR} \delta 14.0,22.6,26.1,29.2,29.3$, $29.4,31.8,59.4,68.2,71.4,72.2,115.0,121.8,126.6,126.9$, $127.7,128.4,130.1,131.4,131.8,146.2,154.8,159.6$, 164.6, 165.3; Anal. calc. for $\mathrm{C}_{33} \mathrm{H}_{40} \mathrm{O}_{7}:$ C $72.24, \mathrm{H} 7.35$; found: C 72.26, $\mathrm{H} 7.35$.

8b (8C-Et): Yield $88 \%$; 'H NMR $\delta 0.87$ (t, 3H, $J=7.0$ $\mathrm{Hz}), 1.19(\mathrm{t}, 6 \mathrm{H}, J=7.0 \mathrm{~Hz}), 1.20-1.84(\mathrm{~m}, 12 \mathrm{H}), 3.49-3.63$ $(\mathrm{m}, 4 \mathrm{H}), 3.71(\mathrm{~d}, 4 \mathrm{H}, J=5.1 \mathrm{~Hz}), 4.01(\mathrm{t}, 2 \mathrm{H}, J=6.6 \mathrm{~Hz})$, 5.36 (quin, $1 \mathrm{H}, J=5.1 \mathrm{~Hz}), 7.00(\mathrm{~d}, 2 \mathrm{H}, J=8.8 \mathrm{~Hz}), 7.31(\mathrm{~d}$, $2 \mathrm{H}, J=8.8 \mathrm{~Hz}), 7.60(\mathrm{~d}, 2 \mathrm{H}, J=8.8 \mathrm{~Hz}), 7.65(\mathrm{~d}, 2 \mathrm{H}, J=8.4$ $\mathrm{Hz}), 8.16(\mathrm{~d}, 2 \mathrm{H}, J=8.8 \mathrm{~Hz}), 8.23(\mathrm{~d}, 2 \mathrm{H}, J=8.4 \mathrm{~Hz}) ;{ }^{13} \mathrm{C}$ NMR $\delta 14.1,15.1,22.6,26.0,29.2,29.3,29.4,31.7,66.8$, $68.0,69.0,72.4,114.9,121.7,126.5,126.9,127.8,128.3$, 130.7, 131.3, 131.6, 146.1, 154.6, 159.5, 164.5, 165.2; Anal. calc. for $\mathrm{C}_{33} \mathrm{H}_{44} \mathrm{O}_{7}: \mathrm{C} 72.89, \mathrm{H} 7.69$; found: $\mathrm{C} 72.96, \mathrm{H} 7.78$.

$8 \mathrm{c}$ (8C-Pr): Yield 72\%; ${ }^{1} \mathrm{H}-\mathrm{NMR} \delta 0.90(\mathrm{t}, 9 \mathrm{H}, J=7.2$ $\mathrm{Hz}$ ), 1.29-1.85 (m, 16H), 3.40-3.51 (m, 4H,), 3.72 (d, 4H, $J$ $=5.1 \mathrm{~Hz}$ ), 4.00 (t, $2 \mathrm{H}, J=6.6 \mathrm{~Hz}$ ), 5.38 (quin, $1 \mathrm{H}, J=5.1$ $\mathrm{Hz}), 7.00(\mathrm{~d}, 2 \mathrm{H}, J=8.8 \mathrm{~Hz}), 7.34(\mathrm{~d}, 2 \mathrm{H}, J=8.8 \mathrm{~Hz}), 7.60$ $(\mathrm{d}, 2 \mathrm{H}, J=8.8 \mathrm{~Hz}), 7.69$ (d, $2 \mathrm{H}, J=8.4 \mathrm{~Hz}), 8.15$ (d, $2 \mathrm{H}, J=$ $8.8 \mathrm{~Hz}), 8.23(\mathrm{~d}, 2 \mathrm{H}, J=8.4 \mathrm{~Hz}) ;{ }^{13} \mathrm{C}-\mathrm{NMR} \delta 10.5,14.0$, $22.6,22.8,26.1,29.2,29.3,29.4,31.8,68.2,69.3,72.6,73.3$, $115.1,121.7,126.6,127.1,128.0,128.4,130.8,131.4$, $131.9,146.3,154.8,159.7,164.6,165.4$; Anal. calc. for $\mathrm{C}_{37} \mathrm{H}_{48} \mathrm{O}_{7}: \mathrm{C} 73.48, \mathrm{H} 8.00$; found: $\mathrm{C} 73.74, \mathrm{H} 8.01$.

8d (8C-Bu): Yield 91\%; 'H-NMR $\delta 0.90$ (t, $9 \mathrm{H}, J=7.0$ $\mathrm{Hz}), 1.29-1.88(\mathrm{~m}, 20 \mathrm{H}), 3.45-3.58(\mathrm{~m}, 4 \mathrm{H}), 3.70(\mathrm{~d}, 4 \mathrm{H}, J=$ $5.1 \mathrm{~Hz}$ ), $4.00(\mathrm{t}, 2 \mathrm{H}, J=6.6 \mathrm{~Hz}$ ), 5.36 (quin, $1 \mathrm{H}, J=5.1 \mathrm{~Hz}$ ), $7.00(\mathrm{~d}, 2 \mathrm{H}, J=8.8 \mathrm{~Hz}), 7.31(\mathrm{~d}, 2 \mathrm{H}, J=8.6 \mathrm{~Hz}), 7.60(\mathrm{~d}$, $2 \mathrm{H}, J=8.8 \mathrm{~Hz}), 7.69(\mathrm{~d}, 2 \mathrm{H}, J=8.4 \mathrm{~Hz}), 8.15(\mathrm{~d}, 2 \mathrm{H}, J=8.8$ $\mathrm{Hz}), 8.23\left(\mathrm{~d}, 2 \mathrm{H}, J=8.4 \mathrm{~Hz}\right.$ ); ${ }^{13} \mathrm{C}-\mathrm{NMR} \delta 13.8,14.1,19.2$, $22.6,26.1,29.2,29.3,29.4,31.7,31.8,68.2,69.4,71.4,72.6$, 
$115.1,121.7,126.7,127.1,128.0,128.4,130.8,131.4$, $131.9,146.3,154.8,159.7,164.6,165.4$; Anal. calc. for $\mathrm{C}_{39} \mathrm{H}_{52} \mathrm{O}_{7}: \mathrm{C} 74.02, \mathrm{H} 8.28$; found: C 73.91, H 8.39.

$8 \mathrm{e}\left(8 \mathrm{C}-\mathrm{CH}_{2} \mathrm{CF}_{3}\right)$ : Yield $92 \%$; 'H NMR $\delta 0.90$ (t, $3 \mathrm{H}, J=$ $6.6 \mathrm{~Hz}$ ), $1.32-1.89(\mathrm{~m}, 12 \mathrm{H}), 3.83-4.05(\mathrm{~m}, 10 \mathrm{H}), 5.36$ (quin, $1 \mathrm{H}, J=5.1 \mathrm{~Hz}), 7.01(\mathrm{~d}, 2 \mathrm{H}, J=8.8 \mathrm{~Hz}), 7.34(\mathrm{~d}, 2 \mathrm{H}, J=8.8$ $\mathrm{Hz}), 7.60(\mathrm{~d}, 2 \mathrm{H}, J=8.8 \mathrm{~Hz}), 7.70(\mathrm{~d}, 2 \mathrm{H}, J=8.4 \mathrm{~Hz}), 8.14$ (d, $2 \mathrm{H}, J=8.8 \mathrm{~Hz}), 8.23$ (d, 2H, $J=8.4 \mathrm{~Hz}$ ); ${ }^{13} \mathrm{C}$ NMR $\delta$ $14.4,22.9,26.3,29.4,29.5,29.6,32.1,68.3,68.3,69.0(\mathrm{q}$, $\left.{ }^{2} J_{\mathrm{CP}}=34.3 \mathrm{~Hz}\right), 70.7,71.5,115.3,122.2,124.0\left(\mathrm{q},{ }^{\prime} J_{\mathrm{CP}}=\right.$ $280.0 \mathrm{~Hz}), 126.9,127.1,128.7,131.1,131.7,132.0,146.6$, $155.3,159.9,164.8,165.3$; MS: $\mathrm{m} / \mathrm{z} 684\left(\mathrm{M}^{+}, 1\right), 309(100)$, $196(6), 105(5), 77(6)$.

$8 \mathrm{f}\left(8 \mathrm{C}-\mathrm{CH}_{2} \mathrm{CF}_{2} \mathrm{CF}_{3}\right)$ : Yield $65 \%$; 'H NMR $\delta 0.89(\mathrm{t}, 3 \mathrm{H}, J$ $=6.6 \mathrm{~Hz}), 1.29-1.82(\mathrm{~m}, 12 \mathrm{H}), 3.88-4.04(\mathrm{~m}, 10 \mathrm{H}), 5.35$ (quin, $1 \mathrm{H}, J=5.1 \mathrm{~Hz}), 7.01(\mathrm{~d}, 2 \mathrm{H}, J=8.8 \mathrm{~Hz}), 7.34(\mathrm{~d}, 2 \mathrm{H}$, $J=8.8 \mathrm{~Hz}), 7.60(\mathrm{~d}, 2 \mathrm{H}, J=8.8 \mathrm{~Hz}), 7.70(\mathrm{~d}, 2 \mathrm{H}, J=8.4$ $\mathrm{Hz}), 8.13(\mathrm{~d}, 2 \mathrm{H}, J=8.8 \mathrm{~Hz}), 8.23(\mathrm{~d}, 2 \mathrm{H}, J=8.4 \mathrm{~Hz}),{ }^{13} \mathrm{C}$ NMR $\delta 14.1,22.7,26.0,29.2,29.3,29.4,31.9,68.0\left(\mathrm{t},{ }^{2} J_{\mathrm{Cr}}=\right.$ $26.6 \mathrm{~Hz}), 68.1,70.4,71.1,113.2\left(\mathrm{tq},{ }^{1} J_{\mathrm{Cr}}=255.3 \mathrm{~Hz},{ }^{2} J_{\mathrm{CF}}=\right.$ $37.1 \mathrm{~Hz}), 115.0,118.3\left(\mathrm{qt},{ }^{\prime} J_{\mathrm{Cr}}=286.0,{ }^{2} J_{\mathrm{Cr}}=34.8 \mathrm{~Hz}\right)$, $122.0,126.6,126.8,127.0,128.4,130.8,131.4,131.8$, $146.3,155.0,159.7,164.6,165.0$.

$8 \mathrm{~g}$ ( $9 \mathrm{C}-\mathrm{Me})$ : Yield $97 \%$; 'H NMR $\delta 0.87$ (t, $3 \mathrm{H}, J=6.6$ $\mathrm{Hz}), 1.28-1.84(\mathrm{~m}, 14 \mathrm{H}), 3.40(\mathrm{~s}, 6 \mathrm{H}), 3.67(\mathrm{~d}, 4 \mathrm{H}, J=5.1$ $\mathrm{Hz}$ ), 4.00 (t, $2 \mathrm{H}, J=6.6 \mathrm{~Hz}$ ), 5.39 (quin, $1 \mathrm{H}, J=5 . \mathrm{I} \mathrm{Hz}$ ), $7.00(\mathrm{~d}, 2 \mathrm{H}, J=8.8 \mathrm{~Hz}), 7.31(\mathrm{~d}, 2 \mathrm{H}, J=8.8 \mathrm{~Hz}), 7.59(\mathrm{~d}$, $2 \mathrm{H}, J=8.8 \mathrm{~Hz}), 7.69(\mathrm{~d}, 2 \mathrm{H}, J=8.4 \mathrm{~Hz}), 8.16(\mathrm{~d}, 2 \mathrm{H}, J=8.8$ $\mathrm{Hz}), 8.22\left(\mathrm{~d}, 2 \mathrm{H}, J=8.4 \mathrm{~Hz}\right.$ ). ${ }^{13} \mathrm{C}$ NMR $\delta 14.1,22.6,26.0$, $29.2,29.3,29.4,29.5,31.8,59.3,68.1,71.2,72.0,115.0$, $121.8,126.6,126.9,127.7,128.4,130.1,131.4,131.8$, $146.2,154.8,159.6,164.6,165.3$. Anal. calc. for $\mathrm{C}_{34} \mathrm{H}_{42} \mathrm{O}_{7}$ : C 72.57, H 7.52; found: C 72.73, H 7.58.

8h (9C-Et): Yield $83 \% ;{ }^{1} \mathrm{H}$ NMR $\delta 0.88$ (t, $3 \mathrm{H}, J=7.0$ $\mathrm{Hz}), 1.18(\mathrm{t}, 6 \mathrm{H}, J=7.0 \mathrm{~Hz}), 1.20-1.84(\mathrm{~m}, 14 \mathrm{H}), 3.50-3.58$ $(\mathrm{m}, 4 \mathrm{H}), 3.72(\mathrm{~d}, 4 \mathrm{H}, J=5.1 \mathrm{~Hz}), 4.01(\mathrm{t}, 2 \mathrm{H}, J=6.6 \mathrm{~Hz})$, 5.37 (quin, $1 \mathrm{H}, J=5.1 \mathrm{~Hz}), 7.00(\mathrm{~d}, 2 \mathrm{H}, J=8.8 \mathrm{~Hz}), 7.31$ (d, $2 \mathrm{H}, J=8.8 \mathrm{~Hz}), 7.58(\mathrm{~d}, 2 \mathrm{H}, J=8.8 \mathrm{~Hz}), 7.69(\mathrm{~d}, 2 \mathrm{H}, J=8.4$ $\mathrm{Hz}), 8.14(\mathrm{~d}, 2 \mathrm{H}, J=8.8 \mathrm{~Hz}), 8.22(\mathrm{~d}, 2 \mathrm{H}, J=8.4 \mathrm{~Hz}) ;{ }^{13} \mathrm{C}$ NMR 14.1, 15.1, 22.6, 26.0, 29.2, 29.3, 29.4, 29.5, 31.7, $66.8,68.0,69.0,72.4,114.9,121.7,126.5,126.9,127.8$, $128.3,130.7,131.3,131.6,146.1,154.6,159.5,164.5$, 165.2; Anal. calc. for $\mathrm{C}_{36} \mathrm{H}_{46} \mathrm{O}_{7}: \mathrm{C} 73.19, \mathrm{H} 7.85$; found: $\mathrm{C}$ 73.41, H 7.97.

$8 \mathbf{~ ( 9 C - P r ) : ~ Y i e l d ~ 9 5 \% ; ~ ' H ~ N M R ~} \delta 0.90(\mathrm{t}, 9 \mathrm{H}, J=7.3 \mathrm{~Hz}$ ), $1.28-1.85(\mathrm{~m}, 18 \mathrm{H}), 3.40-3.51(\mathrm{~m}, 4 \mathrm{H}), 3.72$ (d, $4 \mathrm{H}, J=5.1$ $\mathrm{Hz}$ ), 4.00 (t, $2 \mathrm{H}, J=6.6 \mathrm{~Hz}$ ), 5.38 (quin, $1 \mathrm{H}, J=5.1 \mathrm{~Hz}$ ), $7.00(\mathrm{~d}, 2 \mathrm{H}, J=8.8 \mathrm{~Hz}), 7.31(\mathrm{~d}, 2 \mathrm{H}, J=8.8 \mathrm{~Hz}), 7.59(\mathrm{~d}$, $2 \mathrm{H}, J=8.8 \mathrm{~Hz}), 7.69(\mathrm{~d}, 2 \mathrm{H}, J=8.4 \mathrm{~Hz}), 8.15(\mathrm{~d}, 2 \mathrm{H}, J=8.8$ $\mathrm{Hz}), 8.22(\mathrm{~d}, 2 \mathrm{H}, J=8.4 \mathrm{~Hz}) .{ }^{13} \mathrm{C}$ NMR $\delta 10.5,14.1,22.6$, $22.7,26.0,29.2,29.3,29.4,29.5,31.8,68.1,69.2,72.4,73.1$, $114.9,121.7,126.6,126.9,127.8,128.3,130.7,131.3$, $131.7,146.2,154.6,159.6,164.5,165.3$. Anal. calc. for $\mathrm{C}_{38} \mathrm{H}_{50} \mathrm{O}_{7}: \mathrm{C} 73.76, \mathrm{H} 8.14$; found: $\mathrm{C} 73.61, \mathrm{H} 8.28$.

8 j (9C-Bu): Yield 96\%; ${ }^{1} \mathrm{H}$ NMR $\delta 0.90$ (t, 9H, $J=7.0$ $\mathrm{Hz}), 1.29-1.85(\mathrm{~m}, 22 \mathrm{H}), 3.43-3.58(\mathrm{~m}, 4 \mathrm{H}), 3.70(\mathrm{~d}, 4 \mathrm{H}, J=$ $5.1 \mathrm{~Hz}$ ), 4.00 (t, $2 \mathrm{H}, J=6.6 \mathrm{~Hz}$ ), 5.36 (quin, $1 \mathrm{H}, J=5.1 \mathrm{~Hz}$ ), $7.00(\mathrm{~d}, 2 \mathrm{H}, J=8.8 \mathrm{~Hz}), 7.31$ (d, $2 \mathrm{H}, J=8.8 \mathrm{~Hz}), 7.59$ (d, $2 \mathrm{H}, J=8.8 \mathrm{~Hz}), 7.69(\mathrm{~d}, 2 \mathrm{H}, J=8.4 \mathrm{~Hz}), 8.15(\mathrm{~d}, 2 \mathrm{H}, J=8.8$ $\mathrm{Hz}), 8.23\left(\mathrm{~d}, 2 \mathrm{H}, J=8.4 \mathrm{~Hz}\right.$ ). ${ }^{13} \mathrm{C}$ NMR $\delta 13.9,14.1,19.2$, $22.2,26.0,29.2,29.3,29.4,29.5,31.6,31.8,68.1,69.3,71.3$, $72.5,114.9,121.7,126.6,126.9,127.9,128.4,130.8,131.4$, $131.8,146.2,154.6,159.6,164.6,165.3$. Anal. calc. for $\mathrm{C}_{40} \mathrm{H}_{54} \mathrm{O}_{7}: \mathrm{C} 74.27, \mathrm{H}$ 8.41; found: $\mathrm{C} 73.31, \mathrm{H} 8.41$.

8k $\left(9 \mathrm{C}-\mathrm{CH}_{2} \mathrm{CF}_{3}\right)$ : Yield $80 \%$; ${ }^{1} \mathrm{H}$ NMR $\delta 0.89$ (t, $3 \mathrm{H}, J=$ $6.6 \mathrm{~Hz}$ ), $1.28-1.85(\mathrm{~m}, 14 \mathrm{H}), 3.83-4.04(\mathrm{~m}, 10 \mathrm{H}), 5.37$ (quin, $1 \mathrm{H}, J=5.1 \mathrm{~Hz}), 7.00(\mathrm{~d}, 2 \mathrm{H}, J=8.8 \mathrm{~Hz}), 7.34(\mathrm{~d}, 2 \mathrm{H}, J=8.8$ $\mathrm{Hz}$ ), $7.60(\mathrm{~d}, 2 \mathrm{H}, J=8.8 \mathrm{~Hz}$ ), $7.70(\mathrm{~d}, 2 \mathrm{H}, J=8.4 \mathrm{~Hz}), 8.13$ (d, $2 \mathrm{H}, J=8.8 \mathrm{~Hz}), 8.23$ (d, $2 \mathrm{H}, J=8.4 \mathrm{~Hz}) ;{ }^{13} \mathrm{C}$ NMR $\delta$ $14.1,22.7,26.0,29.2,29.3,29.4,29.5,31.9,68.2,68.8(\mathrm{q}$, $\left.{ }^{2} J_{\mathrm{Cr}}=34.3 \mathrm{~Hz}\right), 70.4,71.3,115.0,122.0,124.0\left(\mathrm{q},{ }^{1} J_{\mathrm{CF}}=\right.$ $280.0 \mathrm{~Hz}), 126.7,126.9,127.1,128.4,130.8,131.4,131.8$, 146.3, 155.1, 159.7, 164.0, 165.1; MS: $\mathrm{m} / \mathrm{z} 698\left(\mathrm{M}^{+}, 1\right), 337$ (100), $196(6), 105(3), 77(6)$.

$81\left(9 \mathrm{C}-\mathrm{CH}_{2} \mathrm{CF}_{2} \mathrm{CF}_{3}\right)$ : Yield $90 \% ;{ }^{1} \mathrm{H}$ NMR $\delta 0.89(\mathrm{t}, 3 \mathrm{H}, J$ $=6.6 \mathrm{~Hz}), 1.28-1.81(\mathrm{~m}, 14 \mathrm{H}), 3.88-4.04(\mathrm{~m}, 10 \mathrm{H}), 5.34$ (quin, $1 \mathrm{H}, J=5.1 \mathrm{~Hz}$ ), $7.00(\mathrm{~d}, 2 \mathrm{H}, J=8.8 \mathrm{~Hz}), 7.34(\mathrm{~d}, 2 \mathrm{H}$, $J=8.8 \mathrm{~Hz}), 7.60$ (d, $2 \mathrm{H}, J=8.8 \mathrm{~Hz}), 7.70(\mathrm{~d}, 2 \mathrm{H}, J=8.4$ $\mathrm{Hz}), 8.13(\mathrm{~d}, 2 \mathrm{H}, J=8.8 \mathrm{~Hz}), 8.23(\mathrm{~d}, 2 \mathrm{H}, J=8.4 \mathrm{~Hz}) ;{ }^{13} \mathrm{C}$ NMR $\delta 14.1,22.7,26.0,28.9,29.2,29.4,29.5,31.9,68.0(\mathrm{t}$, $\left.{ }^{2} J_{\mathrm{CF}}=26.6 \mathrm{~Hz}\right), 68.5,70.4,71.4,113.2\left(\mathrm{tq},{ }^{1} J_{\mathrm{CF}}=255.3,{ }^{2} J_{\mathrm{CF}}\right.$ $=37.1 \mathrm{~Hz}), 115.0,118.3\left(\mathrm{qt},{ }^{4} J_{\mathrm{CF}}=286.0,{ }^{2} J_{\mathrm{CF}}=34.8 \mathrm{~Hz}\right)$, $122.0,126.6,126.8,127.0,128.4,130.8,131.4,131.7$, $146.3,155.0,160.0,164.5,165.0$.

$8 \mathrm{~m}$ (10C-Me): Yield 96\%; ${ }^{1} \mathrm{H}-\mathrm{NMR} \delta 0.87$ (t, 3H, $J=6.6$ $\mathrm{Hz}$ ), $1.15-1.84(\mathrm{~m}, 16 \mathrm{H}), 3.39$ (s, 6H), 3.67 (d, $4 \mathrm{H}, J=5.1$ $\mathrm{Hz}$ ), 4.00 (t, $2 \mathrm{H}, J=6.6 \mathrm{~Hz}$ ), 5.38 (quin, $1 \mathrm{H}, J=5.1 \mathrm{~Hz}$ ), $6.97(\mathrm{~d}, 2 \mathrm{H}, J=8.8 \mathrm{~Hz}), 7.33(\mathrm{~d}, 2 \mathrm{H}, J=8.8 \mathrm{~Hz}), 7.58(\mathrm{~d}$, $2 \mathrm{H}, J=8.8 \mathrm{~Hz}), 7.69(\mathrm{~d}, 2 \mathrm{H}, J=8.4 \mathrm{~Hz}), 8.15(\mathrm{~d}, 2 \mathrm{H}, J=8.8$ $\mathrm{Hz}$ ), $8.22\left(\mathrm{~d}, 2 \mathrm{H}, J=8.4 \mathrm{~Hz}\right.$ ); ${ }^{13} \mathrm{C}-\mathrm{NMR} \delta 14.0,22.7,26.0$, $29.2,29.3,29.4,29.5,29.6,31.9,59.3,68.1,71.3,72.0$, $114.9,121.8,126.6,126.9,127.7,128.4,130.8,131.4$, $131.8,146.2,154.7,159.6,164.6,165.3$; Anal. calc. for $\mathrm{C}_{35} \mathrm{H}_{44} \mathrm{O}_{7}: \mathrm{C} 72.89, \mathrm{H} 7.69$; found: $\mathrm{C} 72.65, \mathrm{H} 7.79$.

8n (10C-Et): Yield $90 \% ;{ }^{1} \mathrm{H}$ NMR $\delta 0.87(\mathrm{t}, 3 \mathrm{H}, J=7.0$ $\mathrm{Hz}), 1.19(\mathrm{t}, 6 \mathrm{H}, J=7.0 \mathrm{~Hz}), 1.20-1.84(\mathrm{~m}, 16 \mathrm{H}), 3.50-3.60$ $(\mathrm{m}, 4 \mathrm{H}), 3.71(\mathrm{~d}, 4 \mathrm{H}, J=5.1 \mathrm{~Hz}), 4.01(\mathrm{t}, 2 \mathrm{H}, J=6.6 \mathrm{~Hz})$, 5.36 (quin, $1 \mathrm{H}, J=5.1 \mathrm{~Hz}), 6.99$ (d, $2 \mathrm{H}, J=8.8 \mathrm{~Hz}), 7.30$ (d, $2 \mathrm{H}, J=8.8 \mathrm{~Hz}), 7.58(\mathrm{~d}, 2 \mathrm{H}, J=8.8 \mathrm{~Hz}), 7.69(\mathrm{~d}, 2 \mathrm{H}, J=8.4$ $\mathrm{Hz}), 8.14(\mathrm{~d}, 2 \mathrm{H}, J=8.8 \mathrm{~Hz}), 8.22(\mathrm{~d}, 2 \mathrm{H}, J=8.4 \mathrm{~Hz}) ;{ }^{13} \mathrm{C}$ NMR $\delta 14.1,15.1,22.6,26.0,29.2,29.3,29.4,29.5,29.6$, $31.7,66.8,68.0,69.0,72.4,114.9,121.7,126.5,126.9$, $127.8,128.3,130.7,131.3,131.6,146.1,154.6159 .5,164.5$, 165.2; Anal. calc. for $\mathrm{C}_{37} \mathrm{H}_{48} \mathrm{O}_{7}: \mathrm{C} 73.48, \mathrm{H} 8.00$; found: $\mathrm{C}$ $72.71, \mathrm{H} 8.18$.

80 (10C-Pr): Yield 90\%; 'H-NMR $\delta 0.89$ (t, 3H, $J=7.2$ $\mathrm{Hz}), 1.28-1.88(\mathrm{~m}, 26 \mathrm{H}), 3.38-3.48(\mathrm{~m}, 4 \mathrm{H}), 3.71(\mathrm{~d}, 4 \mathrm{H}, J=$ $5.1 \mathrm{~Hz}), 4.00(\mathrm{t}, 2 \mathrm{H}, J=6.6 \mathrm{~Hz}), 5.37$ (quin, $1 \mathrm{H}, J=5.1 \mathrm{~Hz}$ ), $7.00(\mathrm{~d}, 2 \mathrm{H}, J=8.8 \mathrm{~Hz}), 7.27(\mathrm{~d}, 2 \mathrm{H}, J=8.8 \mathrm{~Hz}), 7.59(\mathrm{~d}$, $2 \mathrm{H}, J=8.8 \mathrm{~Hz}), 7.69(\mathrm{~d}, 2 \mathrm{H}, J=8.4 \mathrm{~Hz}), 8.15(\mathrm{~d}, 2 \mathrm{H}, J=8.8$ $\mathrm{Hz}), 8.22\left(\mathrm{~d}, 2 \mathrm{H}, J=8.4 \mathrm{~Hz}\right.$ ); ${ }^{13} \mathrm{C}-\mathrm{NMR} \delta 10.5,14.1,22.7$, $22.8,26.0,29.2,29.3,29.4,29.5,29.6,31.9,68.1,69.3,72.5$, $73.2,114.9,121.7,126.6,126.9,127.8,128.3,130.7,131.3$, $131.7,146.2,154.6,159.6,164.5,165.3$; Anal. calc. for 
$\mathrm{C}_{39} \mathrm{H}_{52} \mathrm{O}_{7}: \mathrm{C} 74.02, \mathrm{H} 8.28$; found: C 73.99, H 8.39.

$8 p$ (10C-Bu): Yield 75\%; 'H-NMR $\delta 0.89(\mathrm{t}, 9 \mathrm{H}, J=7.0$ $\mathrm{Hz}), 1.29-1.85(\mathrm{~m}, 24 \mathrm{H}), 3.44-3.55(\mathrm{~m}, 4 \mathrm{H}), 3.71(\mathrm{~d}, 4 \mathrm{H}, J=$ $5.1 \mathrm{~Hz}$ ), 3.99 (t, 2H, $J=6.6 \mathrm{~Hz}$ ), 5.38 (quin, $1 \mathrm{H}, J=5.1 \mathrm{~Hz}$ ), 6.99 (d, $2 \mathrm{H}, J=8.8 \mathrm{~Hz}), 7.31$ (d, $2 \mathrm{H}, J=8.8 \mathrm{~Hz}), 7.58$ (d, $2 \mathrm{H}, J=8.8 \mathrm{~Hz}), 7.68(\mathrm{~d}, 2 \mathrm{H}, J=8.4 \mathrm{~Hz}), 8.15(\mathrm{~d}, 2 \mathrm{H}, J=8.8$ $\mathrm{Hz}), 8.21$ (d, 2H, $J=8.4 \mathrm{~Hz}$ ); ${ }^{13} \mathrm{C}-\mathrm{NMR} \delta 13.9,14.1,19.2$, $22.7,26.0,29.2,29.3,29.4,29.5,29.6,31.6,31.9,68.1,69.3$, $71.3,72.5,114.9,121.7,126.6,126.9,127.9,128.4,130.8$, $131.4,131.8,146.2,154.7,159.6,164.6,165.3$; Anal. calc. for $\mathrm{C}_{41} \mathrm{H}_{36} \mathrm{O}_{7}: \mathrm{C} 74.51, \mathrm{H} 8.54$; found: $\mathrm{C} 74.20, \mathrm{H} 8.47$.

$8 \mathrm{q}\left(10 \mathrm{C}-\mathrm{CH}_{2} \mathrm{CF}_{3}\right)$ : Yield $79 \%$; 'H NMR $\delta 0.90(\mathrm{t}, 3 \mathrm{H}, J=$ $6.6 \mathrm{~Hz}), 1.32-1.90(\mathrm{~m}, 16 \mathrm{H}), 3.83-4.05(\mathrm{~m}, 10 \mathrm{H}), 5.36$ (quin, $1 \mathrm{H}, J=5.1 \mathrm{~Hz}), 7.00(\mathrm{~d}, 2 \mathrm{H}, J=8.8 \mathrm{~Hz}), 7.34(\mathrm{~d}, 2 \mathrm{H}$, $J=8.8 \mathrm{~Hz}), 7.60(\mathrm{~d}, 2 \mathrm{H}, J=8.8 \mathrm{~Hz}), 7.70(\mathrm{~d}, 2 \mathrm{H}, J=8.4$ $\mathrm{Hz}$ ), 8.14 (d, $2 \mathrm{H}, J=8.8 \mathrm{~Hz}), 8.23$ (d, $2 \mathrm{H}, J=8.4 \mathrm{~Hz}$ ); ${ }^{13} \mathrm{C}$ NMR $\delta 14.1,22.6,26.0,29.2,29.3,29.4,29.5,29.6,31.9$, $68.2,68.7,68.8\left(\mathrm{q},{ }^{2} J_{\mathrm{CF}}=34.3 \mathrm{~Hz}\right), 70.7,71.3,115.0,122.0$, $124.0\left(\mathrm{q},{ }^{1} J_{\mathrm{Cr}}=280.0 \mathrm{~Hz}\right), 126.6,126.9,127.1,128.4,130.8$, $131.4,131.8,146.3,155.0,159.6,164.5,165.1 ; \mathrm{MS}: \mathrm{m} / \mathrm{z}$ $712\left(\mathrm{M}^{+}, 3\right), 337(100), 196(5), 105(2), 77(3)$.

8r $\left(10 \mathrm{C}-\mathrm{CH}_{2} \mathrm{CF}_{2} \mathrm{CF}_{3}\right)$ : Yield $63 \%$; ${ }^{1} \mathrm{H}$ NMR $\delta 0.88(\mathrm{t}, 3 \mathrm{H}$, $J=6.6 \mathrm{~Hz}), 1.27-1.82(\mathrm{~m}, 16 \mathrm{H}), 3.88-4.04(\mathrm{~m}, 10 \mathrm{H}), 5.35$ (quin, $1 \mathrm{H}, J=5.1 \mathrm{~Hz}), 7.01(\mathrm{~d}, 2 \mathrm{H}, J=8.8 \mathrm{~Hz}), 7.34(\mathrm{~d}, 2 \mathrm{H}$, $J=8.8 \mathrm{~Hz}), 7.60(\mathrm{~d}, 2 \mathrm{H}, J=8.8 \mathrm{~Hz}), 7.70(\mathrm{~d}, 2 \mathrm{H}, J=8.4$ $\mathrm{Hz}$ ), 8.13 (d, $2 \mathrm{H}, J=8.8 \mathrm{~Hz}$ ), 8.23 (d, $2 \mathrm{H}, J=8.4 \mathrm{~Hz}$ ); ${ }^{13} \mathrm{C}$ NMR $\delta 14.1,22.7,26.0,29.2,29.3,29.4,29.5,29.6,31.9$, $68.0\left(\mathrm{t},{ }^{2} J_{\mathrm{CF}}=26.6 \mathrm{~Hz}\right), 68.1,70.4,71.1,113.2\left(\mathrm{tg},{ }^{1} J_{\mathrm{CF}}=\right.$ $\left.255.3 \mathrm{~Hz},{ }^{2} J_{\mathrm{CF}}=37.1 \mathrm{~Hz}\right), 115.0,118.3\left(\mathrm{qt},{ }^{1} J_{\mathrm{CF}}=286.0 \mathrm{~Hz}\right.$, $\left.{ }^{2} J_{\mathrm{CF}}=34.8 \mathrm{~Hz}\right), 122.0,126.6,126.8,127.0,128.4,130.8$, $131.4,131.7,146.3,155.0,159.7,164.5,165.0$.

8s (12C-Me): Yield 96\%; 'H-NMR $\delta 0.87(\mathrm{t}, 3 \mathrm{H}, J=6.6$ $\mathrm{Hz}), 1.23-1.81(\mathrm{~m}, 20 \mathrm{H}), 3.40(\mathrm{~s}, 6 \mathrm{H}), 3.67(\mathrm{~d}, 4 \mathrm{H}, J=5.1$ $\mathrm{Hz}$ ), 4.00 (t, $2 \mathrm{H}, J=6.6 \mathrm{~Hz}$ ), 5.39 (quin, $1 \mathrm{H}, J=5.1 \mathrm{~Hz}$ ), $7.00(\mathrm{~d}, 2 \mathrm{H}, J=8.8 \mathrm{~Hz}), 7.31(\mathrm{~d}, 2 \mathrm{H}, J=8.8 \mathrm{~Hz}), 7.59$ (d, $2 \mathrm{H}, J=8.8 \mathrm{~Hz}), 7.70(\mathrm{~d}, 2 \mathrm{H}, J=8.4 \mathrm{~Hz}), 8.16(\mathrm{~d}, 2 \mathrm{H}, J=8.8$ $\mathrm{Hz}$ ), 8.22 (d, 2H, $J=8.4 \mathrm{~Hz}$ ); ${ }^{13} \mathrm{C}-\mathrm{NMR} \delta 14.0,22.6,26.0$, $29.2,29.3,29.4,29.5,29.6,29.7,30.0,31.9,59.3,68.2,71.3$, $72.1,115.0,121.7,126.6,127.1,127.8,128.4,130.8,131.4$, $131.8,146.3,154.8,159.7,164.5,165.3$; Anal. calc. for $\mathrm{C}_{37} \mathrm{H}_{48} \mathrm{O}_{7}: \mathrm{C} 73.48, \mathrm{H} 8.00$; found: $\mathrm{C} 73.50, \mathrm{H} 8.12$.

8 t (12C-Et): Yield 98\%; ${ }^{1} \mathrm{H}$ NMR $\delta 0.87(\mathrm{t}, 3 \mathrm{H}, J=7.0$ $\mathrm{Hz}), 1.19(\mathrm{t}, 6 \mathrm{H}, J=7.0 \mathrm{~Hz}), 1.20-1.88(\mathrm{~m}, 20 \mathrm{H}), 3.40-3.60$ (m, 4H), 3.72 (d, 4H, $J=5.1 \mathrm{~Hz}$ ), 3.99 (t, $2 \mathrm{H}, J=6.6 \mathrm{~Hz}$ ), 5.36 (quin, $1 \mathrm{H}, J=5.1 \mathrm{~Hz}$ ), $6.99(\mathrm{~d}, 2 \mathrm{H}, J=8.8 \mathrm{~Hz}), 7.31$ (d, $2 \mathrm{H}, J=8.8 \mathrm{~Hz}), 7.58(\mathrm{~d}, 2 \mathrm{H}, J=8.8 \mathrm{~Hz}), 7.68(\mathrm{~d}, 2 \mathrm{H}, J=8.4$ $\mathrm{Hz}), 8.15$ (d, 2H, $J=8.8 \mathrm{~Hz}), 8.22(\mathrm{~d}, 2 \mathrm{H}, J=8.4 \mathrm{~Hz}) ;{ }^{13} \mathrm{C}$ NMR $\delta 14.1,15.1,22.6,26.0,29.2,29.3,29.4,29.5,29.6$, $29.7,30.0,31.7,66.8,68.0,69.0,72.4,114.9,121.7,126.5$, $126.9,127.8,128.3,130.7,131.3,131.6,146.1,154.6$, $159.5,164.5,165.2$; Anal. calc. for $\mathrm{C}_{39} \mathrm{H}_{32} \mathrm{O}_{7}: \mathrm{C} 74.02$, H 8.28; found: C 73.95, H 8.34.

8u (12C-Pr): Yield $85 \% ;{ }^{1} \mathrm{H}-\mathrm{NMR} \delta 0.89(\mathrm{t}, 3 \mathrm{H}, J=7.2$ $\mathrm{Hz}), 1.23-1.84(\mathrm{~m}, 28 \mathrm{H}), 3.40-3.49(\mathrm{~m}, 4 \mathrm{H}), 3.71(\mathrm{~d}, 4 \mathrm{H}, J=$ $5.1 \mathrm{~Hz}), 4.00(\mathrm{t}, 2 \mathrm{H}, J=6.6 \mathrm{~Hz}), 5.37$ (quin, $1 \mathrm{H}, J=5.1 \mathrm{~Hz}$ ), $7.00(\mathrm{~d}, 2 \mathrm{H}, J=8.8 \mathrm{~Hz}), 7.31(\mathrm{~d}, 2 \mathrm{H}, J=8.8 \mathrm{~Hz}), 7.59$ (d, $2 \mathrm{H}, J=8.8 \mathrm{~Hz}), 7.69(\mathrm{~d}, 2 \mathrm{H}, J=8.4 \mathrm{~Hz}), 8.15(\mathrm{~d}, 2 \mathrm{H}, J=8.8$
$\mathrm{Hz}$ ), 8.22 (d, 2H, $J=8.4 \mathrm{~Hz}$ ); ${ }^{13} \mathrm{C}-\mathrm{NMR} \delta 10.5,14.1,22.7$, $22.8,26.0,29.2,29.3,29.4,29.5,29.6,29.7,30.0,31.9,68.2$, $69.3,72.6,73.2,115.1,121.7,126.6,127.1,128.0,128.4$, $130.8,131.4,131.9,146.3,154.8,159.7,164.6,165.3$; Anal. calc. for $\mathrm{C}_{41} \mathrm{H}_{56} \mathrm{O}_{7}: \mathrm{C} 74.51, \mathrm{H} 8.54$; found: $\mathrm{C} 74.72, \mathrm{H} 8.63$.

8w (12C-Bu): Yield 94\%; 'H-NMR $\delta 0.84-1.88(\mathrm{~m}, 36 \mathrm{H})$, $3.40-3.52(\mathrm{~m}, 4 \mathrm{H}), 3.70(\mathrm{~d}, 4 \mathrm{H}, J=5.1 \mathrm{~Hz}), 4.01(\mathrm{t}, 2 \mathrm{H}, J=$ $6.6 \mathrm{~Hz}$ ), 5.37 (quin, $1 \mathrm{H}, J=5.1 \mathrm{~Hz}$ ), 7.00 (d, $2 \mathrm{H}, J=8.8$ $\mathrm{Hz}$ ), 7.31 (d, 2H, $J=8.8 \mathrm{~Hz}$ ), 7.58 (d, $2 \mathrm{H}, J=8.8 \mathrm{~Hz}$ ), 7.69 (d, $2 \mathrm{H}, J=8.4 \mathrm{~Hz}), 8.15$ (d, $2 \mathrm{H}, J=8.8 \mathrm{~Hz}), 8.23$ (d, 2H, $J=$ $8.4 \mathrm{~Hz}) ;{ }^{13} \mathrm{C}-\mathrm{NMR} \delta 13.9,14.1,19.2,22.7,26.0,29.2,29.3$, $29.4,29.5,29.6,29.7,30.0,31.7,31.9,68.2,69.4,71.4,72.7$, $115.1,121.7,126.6,127.1,128.0,128.4,130.8,131.4$, $131.9,146.3,154.8,159.7,164.6,165.3$; Anal. calc. for $\mathrm{C}_{43} \mathrm{H}_{60} \mathrm{O}_{7}: \mathrm{C} 74.97, \mathrm{H} 8.78$; found: $\mathrm{C} 75.12, \mathrm{H} 8.83$.

$8 \mathrm{x}\left(12 \mathrm{C}-\mathrm{CH}_{2} \mathrm{CF}_{3}\right)$ : Yield $62 \% ;{ }^{1} \mathrm{H}$ NMR $\delta 0.90(\mathrm{t}, 3 \mathrm{H}, J=$ $6.6 \mathrm{~Hz}$ ), $1.32-1.90(\mathrm{~m}, 20 \mathrm{H}), 3.83-4.05(\mathrm{~m}, 10 \mathrm{H}$ ), 5.36 (quin, $1 \mathrm{H}, J=5.1 \mathrm{~Hz}), 7.00(\mathrm{~d}, 2 \mathrm{H}, J=8.8 \mathrm{~Hz}), 7.34(\mathrm{~d}, 2 \mathrm{H}, J=8.8$ $\mathrm{Hz}$ ), 7.60 (d, 2H, $J=8.8 \mathrm{~Hz}$ ), 7.70 (d, $2 \mathrm{H}, J=8.4 \mathrm{~Hz}$ ), 8.14 (d, $2 \mathrm{H}, J=8.8 \mathrm{~Hz}), 8.23$ (d, $2 \mathrm{H}, J=8.4 \mathrm{~Hz}$ ); ${ }^{13} \mathrm{C}$ NMR $\delta$ $14.1,22.7,26.0,29.2,29.3,29.4,29.57,29.59,29.6,29.7$, $31.6,68.2,68.9\left(\mathrm{q},{ }^{2} J_{\mathrm{CF}}=34.3 \mathrm{~Hz}\right), 70.4,71.3,115.0,122.0$, $124.0\left(\mathrm{q},{ }^{1} J_{\mathrm{CF}}=280.0 \mathrm{~Hz}\right), 126.7,126.9,127.1,128.4,130.8$, $131.4,131.8,146.3,155.1,159.7,164.6,165.1 ; \mathrm{MS}: \mathrm{m} / \mathrm{z}$ $740\left(\mathrm{M}^{+}, 3\right), 365(100), 196(5), 105(1), 77(2)$.

$8 y\left(12 \mathrm{C}-\mathrm{CH}_{2} \mathrm{CF}_{2} \mathrm{CF}_{3}\right)$ : Yield $75 \%$; ${ }^{1} \mathrm{H}$ NMR $\delta 0.88(\mathrm{t}, 3 \mathrm{H}$, $J=6.6 \mathrm{~Hz}), 1.26-1.82(\mathrm{~m}, 20 \mathrm{H}), 3.88-4.04(\mathrm{~m}, 10 \mathrm{H}), 5.35$ (quin, $1 \mathrm{H}, J=5.1 \mathrm{~Hz}$ ), 7.01 (d, $2 \mathrm{H}, J=8.8 \mathrm{~Hz}), 7.34(\mathrm{~d}, 2 \mathrm{H}$, $J=8.8 \mathrm{~Hz}), 7.60(\mathrm{~d}, 2 \mathrm{H}, J=8.8 \mathrm{~Hz}), 7.70(\mathrm{~d}, 2 \mathrm{H}, J=8.4$ $\mathrm{Hz}$ ), 8.13 (d, $2 \mathrm{H}, J=8.8 \mathrm{~Hz}$ ), 8.23 (d, $2 \mathrm{H}, J=8.4 \mathrm{~Hz}$ ); ${ }^{13} \mathrm{C}$ NMR $\delta 14.1,22.7,26.0,29.2,29.3,29.4,29.5,29.60,29.65$, $29.7,31.9,68.0\left(\mathrm{t},{ }^{2} J_{\mathrm{Cr}}=26.6 \mathrm{~Hz}\right), 68.1,70.4,71.1,113.2$ $\left(\mathrm{tq},{ }^{1} J_{\mathrm{CF}}=255.3 \mathrm{~Hz},{ }^{2} J_{\mathrm{CF}}=37.1 \mathrm{~Hz}\right), 115.0,118.3\left(\mathrm{qt},{ }^{1} J_{\mathrm{CF}}=\right.$ $\left.286.0 \mathrm{~Hz},{ }^{2} J_{\mathrm{CF}}=34.8 \mathrm{~Hz}\right), 122.0,126.6,126.8,127.0,128.4$, $130.8,131.4,131.7,146.3,155.0,159.7,164.5,165.0$.

\section{Results and Discussion}

New achiral swallow-tailed liquid crystals derived from 1,3-dialkoxy-2-propanols, where alkyl is methyl, ethyl, propyl, butyl, $\mathrm{CH}_{2} \mathrm{CF}_{3}$, and $\mathrm{CH}_{2} \mathrm{CF}_{2} \mathrm{CF}_{3}$, were prepared and their mesomorphic properties were investigated.

1,3-Dialkoxy-2-propanols 3 were prepared from the reaction of epichlorohydrin 1 with 2.0 equivalents of sodium alkoxide 2 . Preparation of the final products $\mathbf{8}$ from the alcohols 3 was achieved by the routine synthetic sequences (Scheme 1). The structures of the final products and intermediates were identified by ${ }^{1} \mathrm{H} \mathrm{NMR},{ }^{13} \mathrm{C} N M R$, elemental analysis, and mass spectroscopy.

The mesophase transition temperatures and enthalpies of the compounds 8 (nC-R) were determined by differential scanning calorimetry (DSC) in conjunction with optical polarizing microscopy. Mesophases were identified by observing the microscopic textures of the materials layered between two untreated glass plates. Texture observations at a rate of $0.1^{\circ} \mathrm{C} / \mathrm{min}$ on cooling exhibited that liquid crystals have fairly rich mesophases. 

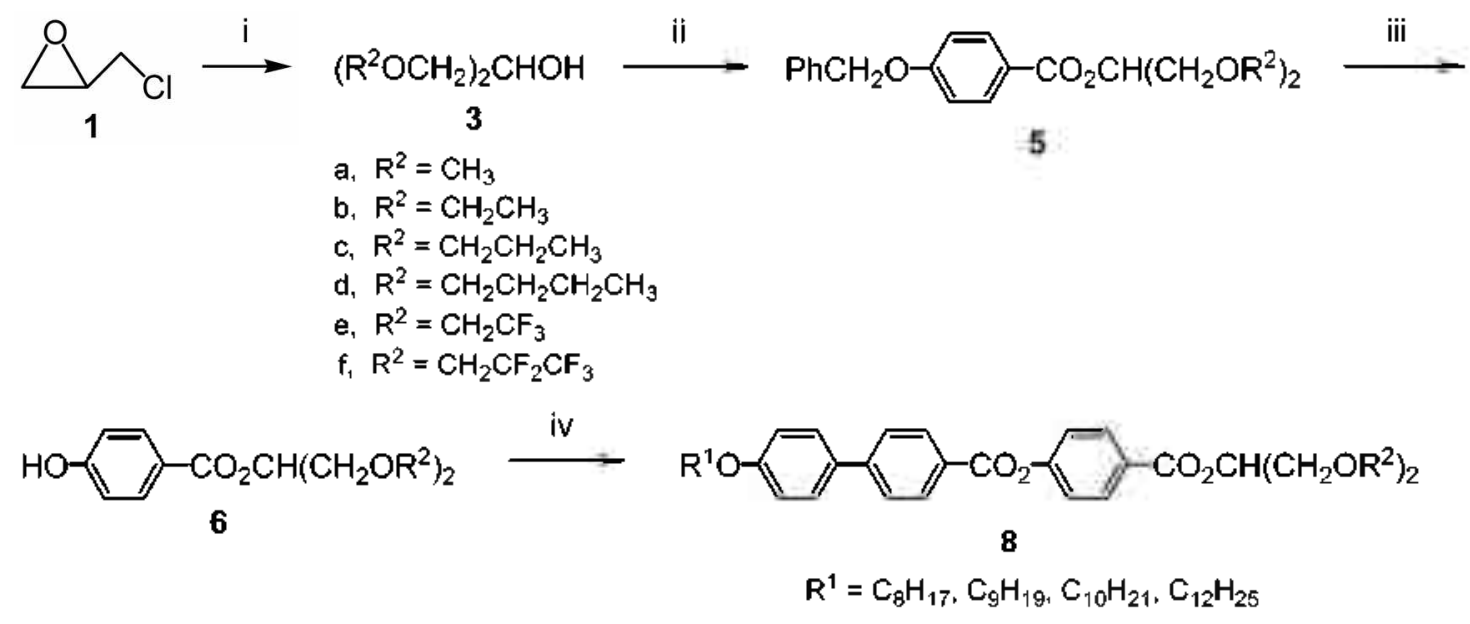

i. $2 \mathrm{R}^{2} \mathrm{ONa}(2)$ ii. $\mathrm{PhCH}_{2} \mathrm{O}-\mathrm{CO}_{2} \mathrm{H}(4), \mathrm{DCC}$

iii. $\mathrm{H}_{2}, \mathrm{Pd} / \mathrm{C}$ iv. $\mathrm{R}^{\prime} \mathrm{O}$

Scheme 1

The SmA phase was characterized by the formation of batomets and focal-conic fan texture and SmC phase by the formation of broken focal-conic fan texture. The SmCalt phase display a striated focal-conic texture in the thicker sample region and it was further characterized by the schlieren texture with two and four singularities in the thinner sample region. ${ }^{3 d}$ The appearance of both of two and four brush singularities in the schlieren texture is diagnostic for the presence of antiferroelectric phase. ${ }^{38}$ The schlieren texture of the SmCalt phase of $\mathbf{8 n}(10 \mathrm{C}-\mathrm{Et})$, which has decy] and ethyl groups in place of $\mathrm{R}^{1}$ and $\mathrm{R}^{2}$, is shown in Figure 1 .

The resulting mesophases and their corresponding phase transition temperatures measured by DSC and microscopic observations are summarized in Table 1. Melting point indicates the temperature at which a mesophase starts to appear on heating.

A plot of phase transition temperature versus substituent $R$ group in the swallow-tail for the compounds 4-(4'-octyloxybiphenyl-4-carbonyloxy)benzoates $8 \mathbf{a}-8 \mathrm{f}$ (8C-R) on cooling is shown in Figure 2. Materials $\mathbf{8 a}$ and $\mathbf{8 d}$ did not show a striated focal-conic or schlieren texture of the SinCalt phase. They exhibited an enantiotropic phase sequences of I-SmASmC-Cr. In contrast to the antiferroelectric ordering of the compound $\mathbf{A}$ containing a branched alkyl group as a swallowtail $^{3 \mathrm{k}}$ the analogous compound $8 \mathrm{a}(8 \mathrm{C}-\mathrm{Me})$ did not exhibit the SmCalt phase.

The difference in compound $\mathbf{A}$ and $\mathbf{8} \mathbf{a}$ might be resulted not from the different steric interactions but from the polar effect of oxygen atoms in the swallow-tail. Because the preferred comformation and all other conformational analysis were reported to be strikingly preserved, when a methylene group in a molecule was replaced with an ethereal oxygen.

However, the compounds $8 \mathrm{~b}(8 \mathrm{C}-\mathrm{Et})$ and $8 \mathrm{c}(8 \mathrm{C}-\mathrm{Pr})$ displayed an 'antiferroelectric-like' SmCalt phase with enantiotropic phase sequences of I-SmA-SmC-SmCalt-Cr. These results indicate that the clinicity is sensitively affected by the molecular structure. The compounds carrying fluorin-

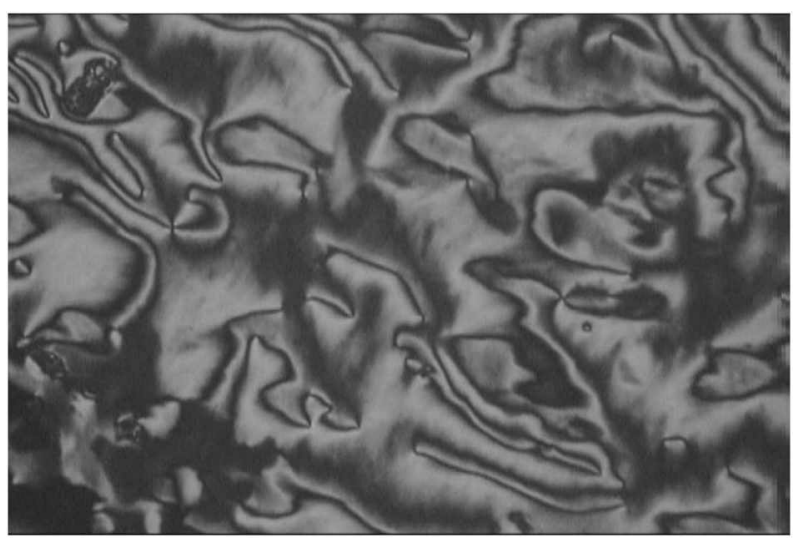

Figure 1. Schliern texture of SmCalt phase of compound $8 \mathbf{n}$ (10C-Et) at $71^{\circ} \mathrm{C}$.

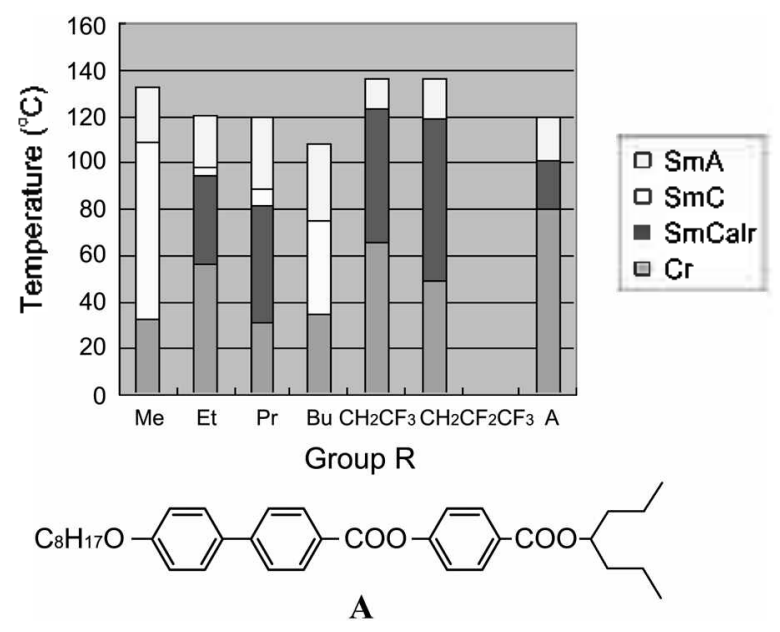

Figure 2. The diagram of the phase sequence and transition temperature for compounds $8 \mathrm{a}-\mathbf{f}(8 \mathrm{C}-\mathrm{R})$ and $\mathbf{A}$.

ated swallow-tails $8 \mathrm{e}\left(8 \mathrm{C}-\mathrm{CH}_{2} \mathrm{CF}_{3}\right)$ and $8 \mathrm{f}\left(8 \mathrm{C}-\mathrm{CH}_{2} \mathrm{CF}_{2} \mathrm{CF}_{3}\right)$ were found to exhibit SmCalt phases with direct SmA- 
Table 1. Phase transition temperatures $\left({ }^{\circ} \mathrm{C}\right)$ and enthalpies $\left(\Delta H / \mathrm{kJmol}^{-1}\right)($ in italics) for compounds 8 on cooling

\begin{tabular}{|c|c|c|c|c|c|c|c|c|c|c|}
\hline Compound & $\mathrm{Cr}$ & & SmCalt & & Smc & & $\overline{\mathrm{SmA}}$ & & $\mathrm{I}$ & $\mathrm{mp}$ \\
\hline $8 \mathrm{a}(8 \mathrm{C}-\mathrm{Me})$ & • & $\begin{array}{l}32.9 \\
4.57\end{array}$ & - & & - & $\begin{array}{c}109.2 \\
0.10\end{array}$ & - & $\begin{array}{c}133.1 \\
4.68\end{array}$ & - & 96.5 \\
\hline $\mathbf{8 b}(8 \mathrm{C}-\mathrm{Et})$ & - & $\begin{array}{l}56.6 \\
19.5\end{array}$ & - & $\begin{array}{l}94.4 \\
0.10\end{array}$ & - & $\begin{array}{l}98.0 \\
0.15\end{array}$ & - & $\begin{array}{c}120.8 \\
4.05\end{array}$ & - & 84.3 \\
\hline $8 c(8 \mathrm{C}-\mathrm{Pr})$ & - & $\begin{array}{l}30.9 \\
10.0\end{array}$ & • & $\begin{array}{l}81.9 \\
0.04\end{array}$ & - & $\begin{array}{l}89.5 \\
0.02\end{array}$ & - & $\begin{array}{c}119.7 \\
3.99\end{array}$ & - & 67.2 \\
\hline $8 \mathbf{d}(8 \mathrm{C}-\mathrm{Bu})$ & - & $\begin{array}{l}34.7 \\
13.1\end{array}$ & - & & - & $\begin{array}{l}75.6 \\
0.09\end{array}$ & - & $\begin{array}{c}108.2 \\
3.27\end{array}$ & - & 57.2 \\
\hline $8 \mathrm{e}\left(8 \mathrm{C}-\mathrm{CH}_{2} \mathrm{CF}_{3}\right)$ & - & $\begin{array}{l}65.3 \\
20.4\end{array}$ & - & $\begin{array}{c}123.2 \\
0.14\end{array}$ & - & & - & $\begin{array}{c}136.8 \\
4.89\end{array}$ & - & 88.9 \\
\hline $8 \mathbf{f}\left(8 \mathrm{C}-\mathrm{CH}_{2} \mathrm{CF}_{2} \mathrm{CF}_{3}\right)$ & - & $\begin{array}{l}49.1 \\
1.17\end{array}$ & - & $\begin{array}{c}119.2 \\
0.29\end{array}$ & - & & - & $\begin{array}{c}136.3 \\
4.08\end{array}$ & - & 63.8 \\
\hline $8 g(9 \mathrm{C}-\mathrm{Me})$ & - & $\begin{array}{l}48.9 \\
13.9\end{array}$ & - & & - & $\begin{array}{l}97.8 \\
0.07\end{array}$ & - & $\begin{array}{c}122.5 \\
3.66\end{array}$ & - & 86.3 \\
\hline $\mathbf{8 h}(9 \mathrm{C}-\mathrm{Et})$ & • & $\begin{array}{l}48.0 \\
14.5\end{array}$ & • & $\begin{array}{l}88.4 \\
1.14\end{array}$ & • & $\begin{array}{l}91.0 \\
0.13\end{array}$ & • & $\begin{array}{c}110.1 \\
3.25\end{array}$ & - & 75.0 \\
\hline $8 \mathbf{i}(9 \mathrm{C}-\mathrm{Pr})$ & - & $\begin{array}{l}42.2 \\
18.0\end{array}$ & - & & - & $\begin{array}{l}83.0 \\
0.14\end{array}$ & - & $\begin{array}{c}102.2 \\
3.10\end{array}$ & - & 58.0 \\
\hline $8 j(9 C-B u)$ & - & $\begin{array}{l}16.7 \\
13.5\end{array}$ & - & & - & $\begin{array}{l}74.4 \\
0.11\end{array}$ & • & $\begin{array}{l}99.0 \\
2.77\end{array}$ & - & 55.2 \\
\hline $\mathbf{8 k}\left(9 \mathrm{C}-\mathrm{CH}_{2} \mathrm{CF}_{3}\right)$ & - & $\begin{array}{l}60.7 \\
22.4\end{array}$ & - & $\begin{array}{c}110.6 \\
0.02\end{array}$ & - & & • & $\begin{array}{c}122.9 \\
3.32\end{array}$ & - & 84.5 \\
\hline $81\left(9 \mathrm{C}-\mathrm{CH}_{2} \mathrm{CF}_{2} \mathrm{CF}_{3}\right)$ & - & $\begin{array}{l}13.2 \\
11.1\end{array}$ & • & $\begin{array}{c}112.8 \\
0.08\end{array}$ & - & & - & $\begin{array}{c}127.6 \\
2.65\end{array}$ & - & 61.2 \\
\hline $8 \mathbf{m}$ (10C-Me) & • & $\begin{array}{l}50.3 \\
24.8\end{array}$ & - & & - & $\begin{array}{c}105.6 \\
0.13\end{array}$ & - & $\begin{array}{c}123.4 \\
3.88\end{array}$ & - & 92.4 \\
\hline $8 \mathbf{n}(10 \mathrm{C}-\mathrm{Et})$ & - & $\begin{array}{l}36.0 \\
12.5\end{array}$ & - & $\begin{array}{l}76.8 \\
0.02\end{array}$ & - & $\begin{array}{l}97.4 \\
0.40\end{array}$ & - & $\begin{array}{c}105.1 \\
1.49\end{array}$ & - & 76.3 \\
\hline $80(10 \mathrm{C}-\mathrm{Pr})$ & • & $\begin{array}{l}31.3 \\
19.8\end{array}$ & - & & - & $\begin{array}{l}80.2 \\
0.20\end{array}$ & - & $\begin{array}{l}95.7 \\
2.64\end{array}$ & - & 60.9 \\
\hline $8 p(10 C-B u)$ & - & $\begin{array}{l}27.8 \\
25.5\end{array}$ & - & & - & $\begin{array}{l}75.6 \\
0.12\end{array}$ & . & $\begin{array}{l}97.2 \\
3.32\end{array}$ & - & 55.6 \\
\hline $\mathrm{Sq}\left(10 \mathrm{C}-\mathrm{CH}_{2} \mathrm{CF}_{3}\right)$ & - & $\begin{array}{l}64.3 \\
22.0\end{array}$ & - & $\begin{array}{r}111.2 \\
0.04\end{array}$ & - & $\begin{array}{c}113.2 \\
0.36\end{array}$ & • & $\begin{array}{c}118.9 \\
3.28\end{array}$ & - & 86.5 \\
\hline $8 \mathrm{r}\left(10 \mathrm{C}-\mathrm{CH}_{2} \mathrm{CF}_{2} \mathrm{CF}_{3}\right)$ & - & $\begin{array}{l}26.0 \\
11.4\end{array}$ & - & $\begin{array}{c}110.2 \\
0.50\end{array}$ & - & & - & $\begin{array}{c}118.6 \\
2.78\end{array}$ & - & 65.3 \\
\hline $8 \mathrm{~s}(12 \mathrm{C}-\mathrm{Me})$ & - & $\begin{array}{l}62.5 \\
35.7\end{array}$ & - & & - & $\begin{array}{l}95.0 \\
0.2 I\end{array}$ & - & $\begin{array}{c}111.4 \\
3.45\end{array}$ & - & 94.9 \\
\hline $8 t(12 C-E t)$ & - & $\begin{array}{l}46.9 \\
27.4\end{array}$ & - & & • & $\begin{array}{l}87.7 \\
0.13\end{array}$ & - & $\begin{array}{l}96.4 \\
1.23\end{array}$ & - & 78.5 \\
\hline $811(12 \mathrm{C}-\mathrm{Pr})$ & - & $\begin{array}{l}35.6 \\
22.0\end{array}$ & - & & * & $\begin{array}{l}78.0 \\
0.10\end{array}$ & . & $\begin{array}{c}116.6 \\
1.54\end{array}$ & • & 63.8 \\
\hline $8 w(12 C-B u)$ & - & $\begin{array}{l}55.1 \\
40.5\end{array}$ & - & & - & $\begin{array}{l}72.5 \\
0.17\end{array}$ & - & $\begin{array}{l}88.6 \\
1.78\end{array}$ & - & 69.1 \\
\hline $8 \times\left(12 \mathrm{C}-\mathrm{CH}_{2} \mathrm{CF}_{3}\right)$ & - & $\begin{array}{l}64.3 \\
26.1\end{array}$ & - & $\begin{array}{l}95.9 \\
0.02\end{array}$ & - & $\begin{array}{c}102.9 \\
0.55\end{array}$ & - & $\begin{array}{c}107.9 \\
2.92\end{array}$ & - & 89.5 \\
\hline $8 \mathbf{y}\left(12 \mathrm{C}-\mathrm{CH}_{2} \mathrm{CF}_{2} \mathrm{CF}_{3}\right)$ & - & $\begin{array}{l}45.8 \\
15.4\end{array}$ & . & $\begin{array}{c}104.5 \\
0.48\end{array}$ & - & & - & $\begin{array}{c}112.9 \\
2.86\end{array}$ & - & 71.0 \\
\hline
\end{tabular}

$\mathrm{Cr}=$ crystalline phase; SmCalt $=$ antiferroelectric-like smectic $\mathrm{C}$ phase; $\mathrm{SmC}=$ smectic $\mathrm{C}$ phase; $\mathrm{SmA}=$ smectic $\mathrm{A}$ phase; $\mathrm{I}=$ isotropic liquid phase.

SmCalt transitions. The swallow-tailed liquid crystals $\mathbf{8 b}, \mathbf{8 c}$, 8e, and 8 f with bisalkoxy swallow-tails showed SmCalt phases at temperature lower, and temperature range broader than the compound $\mathbf{A}^{3 b}$

The compounds $\mathrm{nC}-\mathrm{Me}, \mathrm{nC}-\mathrm{Pr}$, and $\mathrm{nC}-\mathrm{Bu}$ did not exhibit SmCalt phases except for $8 \mathrm{C}-\operatorname{Pr}(\mathbf{8 c})$. Materials nC-Et were found to exhibit SmCalt phases with phase sequences of ISmA-SmC-SmCalt-Cr except for 12C-Et which showed

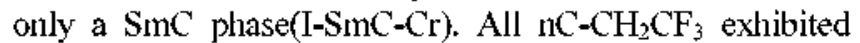
SmCalt phases, and their temperature ranges are broader than those of the corresponding nC-Et. The compounds $8 \mathrm{C}$ $\mathrm{CH}_{2} \mathrm{CF}_{2} \mathrm{CF}_{3}(\mathbf{8 f}), 9 \mathrm{C}-\mathrm{CH}_{2} \mathrm{CF}_{2} \mathrm{CF}_{3}(\mathbf{8 I})$, and $10 \mathrm{C}-\mathrm{CH}_{2} \mathrm{CF}_{2} \mathrm{CF}_{3}$ 
(8r) showed SmCalt phases with direct SmA-SmCalt transitions. Those liquid crystals showed the phase sequences (ISmA-SmCalt-Cr) and exhibited SmCalt phases at temperature lower, and temperature range broader than the compounds with the non-fluorinated swallow-tails. The compound $9 \mathrm{C}-\mathrm{CH}_{2} \mathrm{CF}_{2} \mathrm{CF}_{3}$ (81) exhibited the lowest crystallization temperature $\left(13.2^{\circ} \mathrm{C}\right)$ and the broadest temperature range $\left(99.6^{\circ} \mathrm{C}\right)$ of SmCalt phase among the prepared liquid crystals. The introduction of fluorinated alkyl in swallow-tails causes to raise the melting point, clearing point, and transition temperature for SmCalt phase when compared to the unsubstituted compounds. However, the compounds carrying highly polar fluorine substituted swallow-tails exhibit the more stable SmCalt phases than the analogues with simple alkyl swallow-tails. The effects of the fluoro substituents in the swallow-tails on the determination of clinicity and stability may come from the steric and polar effects since $\mathrm{C}-\mathrm{F}$ bond $(1.38 \AA)$ is slightly longer than that of $\mathrm{C}-\mathrm{H}(1.09 \AA)$ and fluorine is the most electronegative atom. At present, it is not clear how the steric and/or polar interaction do work for the clinicity and stability of the SmCalt phases.

In summary, newly synthesized 4'-(alkyloxy)-[1,1'-biphenyl]-4-carboxylates (nC-R) show diverse phase sequences depending on the $\mathrm{R}$ group in the swallow-tail, which play a major role in determining the clinicity and stability of SmCalt phase. Most of the compounds in the series nC-Me, $\mathrm{nC}-\mathrm{Pr}$, and $\mathrm{nC}-\mathrm{Bu}$ exhibited smectic $\mathrm{C}$ phases synclinic ordering, however, most of the materials $\mathrm{nC}-\mathrm{Et}, \mathrm{nC}-\mathrm{CH}_{2} \mathrm{CF}_{3}$, and $\mathrm{nC}_{-} \mathrm{CH}_{2} \mathrm{CF}_{2} \mathrm{CF}_{3}$ showed SmCalt phases (anticlinic ordering). The clinicity of the compounds with bisalkoxy swallow-tails was found to change sensitively by the molecular structures especially the R group of the swallowtail.

\section{Conclusion}

New achiral swallow-tailed liquid crystals derived from 1,3-dialkoxy-2-propanol were prepared in good yields. These swallow-tailed liquid crystals having 1,3-dialkoxy-2propyl moieties exhibited diverse phase sequences depending on $\mathrm{R}$ groups in the swallow-tails and the length of straight terminal alkyl chains, $\mathrm{n}$. The clinicity and the stability of 'antiferroelectric-like' SmCalt phase in this system largely and sensitively depend upon the R groups. All the compounds carrying the fluorinated alkyl groups in the swallow-tails exhibit SmCalt phase and their temperature ranges are broader than the materials with simple alkyl swallow-tails.

Acknowledgment. This work was performed as part of Advanced Backbone IT Technology Development Project supported by Ministry of Information and Communication in the Republic of Korea.

\section{References}

1. (a) Meyer, R. B.; Liebert, L.; Strzelecki, L.; Keller, P. J. Phys. (France) Lett, 1975, 36, L69. (b) Lee, S. K.; Shin, M. S.; Lee, J. G; Kang, K.-T,; Kim, Y. B, Bull. Korean Chem. Soc, 2004, 25, 1699.

2. Chandani, A. D. L.; Gorecka, E.; Ouchi, Y.; Takezoe, H.; Fukuda, A. Jph. J. Appl. Phss. 1989, 28, L 1265.

3. (a) Nishivama, I.; Goodby, J. W. J. Mater. Chem. 1992, 2, 1015. (b) Ouchi, Y.; Yoshioka, Y.; Ishii, H.; Seki, K.; Kitamura, M.; Noyori, R; Takanishi, Y.; Nishiyama, I. J. Mater. Chem, 1995, 5, 2297. (c) Booth, C. J.; Dunmur, D. A.; Goodby, J. W.; Halely, J.; Toyne, K. J. Lig. Cryst, 1996, 20, 387. (d) Wu, S.-L.; Chiang, C.T. Liq. Cryst. 2002, 29, 39. (e) Tuffin, R. P.; Goodby, J. W.; Bennemann, D.; Heppke, G; Lotzch, D.; Scherowsky, G. Mol. Cryst. Liq. Cryst. 1995, 260,5l.

4. Niori, T.; Sekine, T.; Watanabe, J. Furukawa, T.; Takezoe, H. $J$. Mater. Chem. 1996, 6, 1231 .

5. Nishiyama, I.; Yamamoto, T.; Yamamoto, J.; Goodby, J. W.; Yokoyama, H. J. Mater, Chent. 2003, J3, 1868.

6. (a) Kumazawa, K.; Nakata, M.; Araoka, F.; Takanishi, Y.; Ishikawa, K.; Watanabe, J.; Takezoe, H. J. Moter. Chem. 2004, 14, 157. (b) Lee, S. K.; Heo, S.; Lee, J. G.; Kang, K.-T.; Kumazawa, K.; Nishda, K.; Shimbo, Y.; Takanish, Y.; Watanabe, J.; Doi, T.; Takahashi, T.; Takezoe, H.J. Am. Chem. Soc, 2005, 127, 11085.

7. (a) Wu, S.-L.; Yen, D.-C.; Hsieh, W.J. Liq. Cnst. 1998, 24, 74I. (b) Wu, S.-L.; Hsieh, W.-J. Chent Mater. 1999, II, 852. (c) Wu. S.-L.; Lin, C.-Y. Liq. Cryst. 2003, 30, 205. (d) Wu, S.-L.; Lin, C.Y, Liq. Cryst, 2003, 30, 471, (e) Wu, S.-L.; Chen, F,-D. Liq. Coyst. 2003, 30,991. (f) Wu, S.-L.; Lin, T.-C. Liq. Cryst, 2004, 31, 1469.

8. (a) Gisse, P.; Pavel, J.; Nguyen, H. T.; Lorman, V. L. Feroelectrics 1993, 147, 27. (b) Takanishi, Y.; Takezoe, H.; Fukuda, A.; Komura, H.; Watanabe, J. J. Mater. Chem. 1992, 2, 7I. (c) Takanishi, Y.; Takezoe, H.; Fukuda, A.; Watanabe, J.; Chandani, A. D. L.; Ouchi, Y.; Takezoe, H.; Fukuda, A.; Terashima, K.; Furukawa, K.; Kishi, A.Jpn. J. Appl. Phys. 1989, 28, L1261.

9. Dale, J. Tetrahedron 1974, 30, 1683. 